\title{
IVS - A SECOND GENERATION SPACE VLBI MISSION
}

\author{
GÖRAN PILBRATT
}

European Space Agency Astrophysics Division, ESTEC/SA, Postbus 299, NL-2200 AG Noordwijk, The Netherlands

\begin{abstract}
The International VLBI Satellite is under assessment study by the European Space Agency and the USSR Academy of Sciences. The IVS mission is conceived as a second generation space VLBI mission with the goal of providing higher sensitivity, greater maximum resolution and a wider spectral coverage compared to the precursor missions and, in addition, some single-dish capability.
\end{abstract}

\section{INTRODUCTION}

The International VLBI Satellite (IVS) is a proposal for an orbiting radio telescope intended primarily for very long baseline interferometry (VLBI), but, in addition, also for some single-dish spectral line and cosmic microwave background (CMB) observations.

In its VLBI rôle it will be a second-generation mission offering improved sensitivity, a frequency coverage extending from 5 to $90 \mathrm{GHz}$, or, if feasible, to $220 \mathrm{GHz}$, accurate orbit determination and capability to change orbit. In its single-dish rôle it will enable spectral line observations primarily of molecular oxygen to be performed and also enable accurate high resolution mapping of the CMB decrement towards galaxy clusters to be made.

IVS is ambitious, it is too complex and costly for a single space agency to undertake and is therefore, from its inception, a collaborative undertaking between the European Space Agency (ESA) and the USSR Academy of Sciences (AcdSci) with potential for NASA and other additional participation.

\section{SCIENTIFIC OBJECTIVES}

The scientific objectives span a wide range of topics from the study of molecular clouds including star formation, the physics of masers and stars, galactic and extragalactic distance determinations, the physics of normal and active galactic nuclei (AGNs), including phenomena associated with AGNs such as jets and superluminal motion. Finally some cosmological questions can be addressed. It is convenient to divide the scientific objectives into two groups, namely, those related to VLBI on the one hand, and those related to single-dish observations on the other. 
Continuum, Spectral Line and Polarisation VLBI Science Goals

Compared to the exploratory space VLBI missions RADIOASTRON and VSOP, the IVS mission will be a major advance by virtue of its much greater sensitivity, greater maximum angular resolution and new observing frequencies. The mission will provide a space-based VLBI element with performance comparable to that of those in one of the modern generation of ground-based arrays. This means that not only can great improvements be achieved in "classsical" areas of VLBI but also that exciting new opportunities will open up. The list of scientific objectives include:

(i) The nuclei of galaxies and quasars - the "classical" area of VLBI where new insights can be obtained on the mechanisms of energy release, transport, collimation and dissipation. Tracing changes in source structure through repeated multi-frequency imaging in total intensity and polarisation will be one of the primary tasks of IVS. This should provide unique information on kinematics, magnetic fields, and thermal electron distribution at unprecedented linear resolution.

The capability to image weak radio jets is unique to IVS. This is important since in various "unified schemes" of extragalactic radio sources the variation of the jet/core strength ratio from source to source reflects a wide range of viewing angles of the outflow of the relativistic plasma. Thus, the ability to image weak jets is crucial if jets are to be studied at the full range of viewing angles.

(ii) Radio emitting stars - by imaging these objects for the first time with resolutions often finer than stellar dimensions, significant advances can be expected in understanding of emission mechanisms and magnetic fields. The solar disk at a distance of $10 \mathrm{pc}$ subtends an angular diameter of 1 mas. An observing frequency of $5 \mathrm{GHz}$ is most appropriate for stellar work, the fluxes of stars are weak, ranging from $\mathrm{mJy}$ to tens, possibly hundreds, of $\mathrm{mJy}$; all of this underlines the importance of sensitivity and resolution offered by IVS.

(iii) Cosmic distance scale - by monitoring the motions of extragalactic $\mathrm{H}_{2} \mathrm{O}$ masers, a direct measurement of the cosmic distance scale is possible. However, this technique depends crucially on sensitivity (at $22 \mathrm{GHz}$ ) and requires that the position of IVS can be determined (reconstituted) to an accuracy of a few tens of $\mathrm{cm}$. Using this method the distance to galaxies as far away as in the Virgo cluster could be determined with an accuracy of 10-20\% totally independent of all other methods.

(iv) Observations of extragalactic supernovae remnants can provide new insights into the physics, and also help determining their distances.

(v) Interpretations of images of gravitationally lensed objects rely on the Hubble constant, which, thus, is constrained.

It must also not be forgotten that, as with any telescope breaking new instrumental ground, we can expect much of IVS's contribution to astrophysics to be in discovering unexpected radio structures and in answering as yet unformulated questions.

\section{Single-dish Observations Science Goals}

For a space VLBI mission there will always be some fraction of time when the space segment is idle; thus, a spacecraft with an exciting autonomous capability would enhance the total science return of the mission. The rationale for using IVS as a single-dish radio telescope is two-fold: 
(i) Molecular oxygen $\left(\mathrm{O}_{2}\right)$ has several transitions in the frequency range $56-63 \mathrm{GHz}$, a region of the spectrum which is totally blocked by the Earth's atmosphere. Oxygen is the third most abundant element and is suspected to play a key rôle in the chemical and dynamical evolution of interstellar molecular clouds and star formation regions. Since the formation and destruction of $\mathrm{O}_{2}$ is belived to be fairly well understood, observations of the abundance of $\mathrm{O}_{2}$ would also give abundances of atomic oxygen $(0)$ indirectly.

(ii) IVS would also be very well suited for observations of the CMB decrement (the Sunyaev-Zeldovich effect). Background considerations imply that observations in the $20-80 \mathrm{GHz}$ frequency range are preferable. When observing from a good ground site in the lower part of this range typically $90 \%$ of the data must be discarded because of atmosphere fluctuations. With its large antenna and equipped with a stable wide-band total power backend, IVS could make two-dimensional maps of the decrement towards distant clusters of galaxies. Together with X-ray data these could be used to determine cosmological distances directly and independently.

\section{MISSION REQUIREMENTS}

From the scientific objectives it is clear that the prime mission requirement is that IVS be capable of performing high sensitivity continuum, spectral line and polarisation VLBI observations, and, in addition, single-dish spectral line and CMB total power measurements.

Thus, IVS needs to have a large ( 20 metre class), deployable, accurate $(0.3 / 0.1 \mathrm{~mm}$ rms surface accuracy for $90 / 220 \mathrm{GHz}$ maximum operating frequency) primary reflector. Cooled, state-of-the-art, dual polarisation receivers covering a wide frequency range are required. The frequency bands $4.5-8.5,15-23,42-63$ and $86-120 \mathrm{GHz}$ are foreseen to employ HEMT receivers cooled to $20 \mathrm{~K}$, whereas a $218-222 \mathrm{GHz}$ receiver would be an SIS receiver, requiring cooling to $4 \mathrm{~K}$. Loads at well-determined temperatures are necessary for system performance measurements. For some of the VLBI observations the capability to improve the sensitivity by increasing the coherence time using phase referencing would most be valuable. The single-dish capability requires an onboard spectrometer, a very stable broad-band continuum total power backend, as well as onboard memory for storing the data until they are transmitted to the ground.

The choice of orbit for a space VLBI mission is always a difficult tradeoff to make. Therefore, the intention in the case of IVS is to retain the capability to change orbit during the course of the mission. Three orbits have been studied in some detail. They would all have perigee of $\sim 6000 \mathrm{~km}$, inclination of $63^{\circ}$ and apogees of approximately 20000, 40000 and 150000 $\mathrm{km}$, respectively. The low perigee and high inclination ensure that there is overlap between the shortest space-to-ground and longest ground-to-ground baselines and an improvement in the north-south baseline components for most viewing directions. The maximum resolution attainable increases linearly with maximum baseline length whereas the imaging capability is more difficult to quantify. For the three orbits the size of the synthesized beam at an observing frequency of $43 \mathrm{GHz}$ would be around 50,30 and $10 \mu \mathrm{as}$, respectively, compared to approximately $150 \mu$ as without a space element. The two lower 
orbits would enable high dynamic range imaging to be performed, whereas for the highest orbit the emphasis would not be on imaging.

Although the orbit tolerance is not crucial for IVS (i.e. it is not crucial whether the intended apogee becomes e.g. 20100 instead of $20000 \mathrm{~km}$ ) the orbit reconstitution is. For some scientific objectives the a posteriori position uncertainty requirement is a few tens of $\mathrm{cm}$.

Because of its size and intended high frequency of operation IVS puts stringent demands on its pointing system. The absolute pointing requirement is 10 ", whereas relative pointing and pointing reconstitution must be good to within 5 ". In addition, there is the requirement of real-time contact with a ground tracking station for VLBI observations as well minimizing the restrictions on the pointing direction due to this and other geometrical considerations. Clearly, co-observing VLBI stations on the ground and access to a VLBI correlator are vital.

\section{COLLABORATION AND SCHEDULE}

The baseline for the IVS assessment study assumes collaboration as follows. The spacecraft consists of a service and a payload module. The service module is provided by AcdSci, the payload module, including the radio astronomy antenna, part of the pointing, the radio receiver coolers and the $\mathrm{K}_{u}$-band subsystem, are ESA responsibilities. Launch and flight operations are AcdSci responsibilities while science operations are done by ESA. The phase transfer and science downlink are done by NASA DSN. The scientific payload is provided by national agencies, which is also true for the ground VLBI networks and VLBI correlators.

From an ESA point of view IVS is one of the proposals for the next medium-sized mission (M2) of the long-range Horizon 2000 plan. The proposal was submitted by 74 astronomers in November 1989 and was one of six selected for assessment study in January 1990. This study will be completed in January 1991 and the presentation to the community and selection of a maximum of four proposals for phase A study will take place in March 1991.

The phase A study, which is an industrial study, would commence in January 1992 and last about 14 months, and be followed by a consolidation phase. The recommendations by the ESA advisory bodies and the final selection of the M2 mission would then take place in May/June 1993. After evaluation of replies to an announcement of opportunity the selection of experiments would take place in 1995, the phase B would start in 1996, the phase C/D in 1997, with launch currently foreseen for the year 2001 .

\section{ACKNOWLEDGEMENTS}

The work reported on in this talk is a collective effort involving a large number of individuals in the IVS science team, the European Space Agency, the USSR Academy of Sciences, and the industrial contractors, all of whom deserve credit for their contributions.

The final written version of this paper has benefitted from comments made by Peter Wilkinson and Richard Schilizzi on an earlier draft. 
U. J. Schwarz: What is the planned lifetime of the satellite?

G. Pilbratt: The mission design allows for a lifetime of at least six years.

Peter Dewdney: Are you planning to use relay satellites for telemetry, thus possibly reducing the restrictions on the orientation of the spacecraft at any point in its orbit. These constraints impact u-v coverage, etc.

Goren Pilbratt: No, up to this point we have not considered the use of relay satellites. The use of three onboard telemetry antennas for the Kuband subsystem ensures that communication between IVS and the ground is possible at all times the satellite is visible from a ground station, independent of satellite orientation. 